\title{
Quasi-epitaxial Metal-Halide Perovskite Ligand Shells on PbS Nanocrystals
}

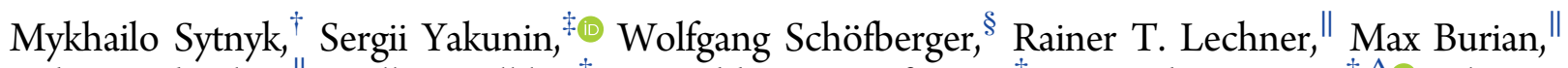

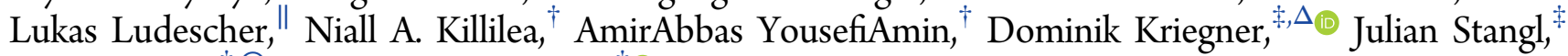
Heiko Groiss, ${ }^{\ddagger}, \mathrm{O}^{\prime}$ and Wolfgang Heiss $*, \dagger$

${ }^{\dagger}$ Materials Science Department (Materials for Electronics and Energy Technology), Friedrich-Alexander Universität Erlangen-Nürnberg, Energy Campus Nürnberg, Nürnberg 90429, Germany

${ }^{\ddagger}$ Institute of Semiconductor and Solid State Physics, Johannes Kepler University Linz, Linz 4040, Austria

${ }^{\S}$ Institute of Organic Chemistry, Johannes Kepler University Linz, Linz 4040, Austria

"Institute of Physics, Montanuniversitaet Leoben, Leoben 8700, Austria

${ }^{\Delta}$ Department of Condensed Matter Physics, Charles University Prague, Prague 12116, Czech Republic

OLaboratory for Electron Microscopy, Karlsruhe Institute of Technology (KIT), Karlsruhe 76128, Germany

Supporting Information

ABSTRACT: Epitaxial growth techniques enable nearly defect free heterostructures with coherent interfaces, which are of utmost importance for high performance electronic devices. While high-vacuum technology-based growth techniques are state-of-the art, here we pursue a purely solution processed approach to obtain nanocrystals with eptaxially coherent and quasi-lattice matched inorganic ligand shells. Octahedral metal-halide clusters, respectively 0-dimensional perovskites, were employed as ligands to match the coordination geometry of the $\mathrm{PbS}$ cubic rock-salt
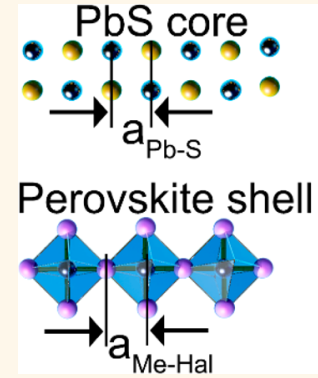

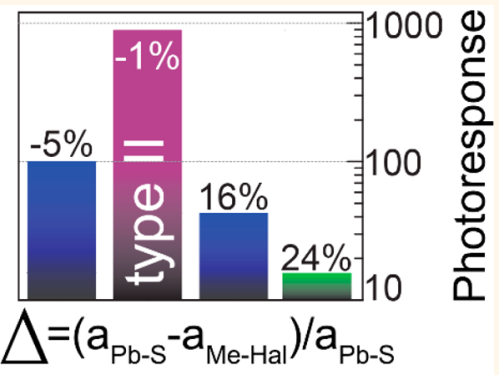
lattice. Different clusters $\left(\mathrm{CH}_{3} \mathrm{NH}_{3}^{+}\right)_{(6-x)}\left[\mathrm{M}^{(x+)} \mathrm{Hal}_{6}\right]^{(6-x)-}$ $\left(\mathrm{M}^{x+}=\mathrm{Pb}(\mathrm{II}), \mathrm{Bi}(\mathrm{III}), \mathrm{Mn}(\mathrm{II})\right.$, In(III), Hal $=\mathrm{Cl}$, I) were attached to the nanocrystal surfaces via a scalable phase transfer procedure. The ligand attachment and coherence of the formed $\mathrm{PbS} /$ ligand core/shell interface was confirmed by combining the results from transmission electron microscopy, small-angle $\mathrm{X}$-ray scattering, nuclear magnetic resonance spectroscopy and powder $\mathrm{X}$-ray diffraction. The lattice mismatch between ligand shell and nanocrystal core plays a key role in performance. In photoconducting devices the best performance (detectivity of $2 \times 10^{11} \mathrm{~cm} \mathrm{~Hz}^{1 / 2} / \mathrm{W}$ with $>110 \mathrm{kHz}$ bandwidth) was obtained with $\left(\mathrm{CH}_{3} \mathrm{NH}_{3}\right)_{3} \mathrm{BiI}_{6}$ ligands, providing the smallest relative lattice mismatch of ca. $-1 \%$. $\mathrm{PbS}$ nanocrystals with such ligands exhibited in millimeter sized bulk samples in the form of pressed pellets a relatively high carrier mobility for nanocrystal solids of $\sim 1.3 \mathrm{~cm}^{2} /(\mathrm{V} \mathrm{s})$, a carrier lifetime of $\sim 70 \mu \mathrm{s}$, and a low residual carrier concentration of $2.6 \times 10^{13} \mathrm{~cm}^{-3}$. Thus, by selection of ligands with appropriate geometry and bond lengths optimized quasi-epitaxial ligand shells were formed on nanocrystals, which are beneficial for applications in optoelectronics.

KEYWORDS: nanocrystals, perovskite, conductive ligands, epitaxy, photodetectors, optoelectronics, semiconductors

$\mathrm{E}$ pitaxy, the deposition of crystalline layers of ordered atoms from gaseous or liquid precursors on top of crystalline substrates, is of major importance in research and industrial semiconductor technology to produce conditioned materials, quantum-structures, and devices with "tailormade" characteristics. ${ }^{1-3}$ Epitaxial materials based on (ultra) high-vacuum technologies benefit from the excellent control over deposition rates, low defect densities, atomically sharp interfaces, precise composition control, and the possibility to fabricate structures of variable dimensionality. ${ }^{1,2}$ Solution-based epitaxial growth has also been achieved, for instance, to obtain oriented nanowires on planar substrates ${ }^{4,5}$ or to overcoat dispersed micro- and nanostructure quasi-substrates with thin functional films ${ }^{6}$ of oriented nanoparticles. ${ }^{7}$ Epitaxial solution growth is widely used today in colloidal chemistry to obtain core/shell structures with various morphologies ${ }^{8-20}$ to reduce surface recombination of photoexcited carriers, ${ }^{8,10,13}$ to tune the attractive interactions between quantum confined electrons and holes, ${ }^{21,22}$ to manipulate nonradiative Auger recombination, ${ }^{23-25}$ or to improve the materials environmental stability. ${ }^{10}$

Received: July 15, 2016

Accepted: January 30, 2017

Published: January 30, 2017 


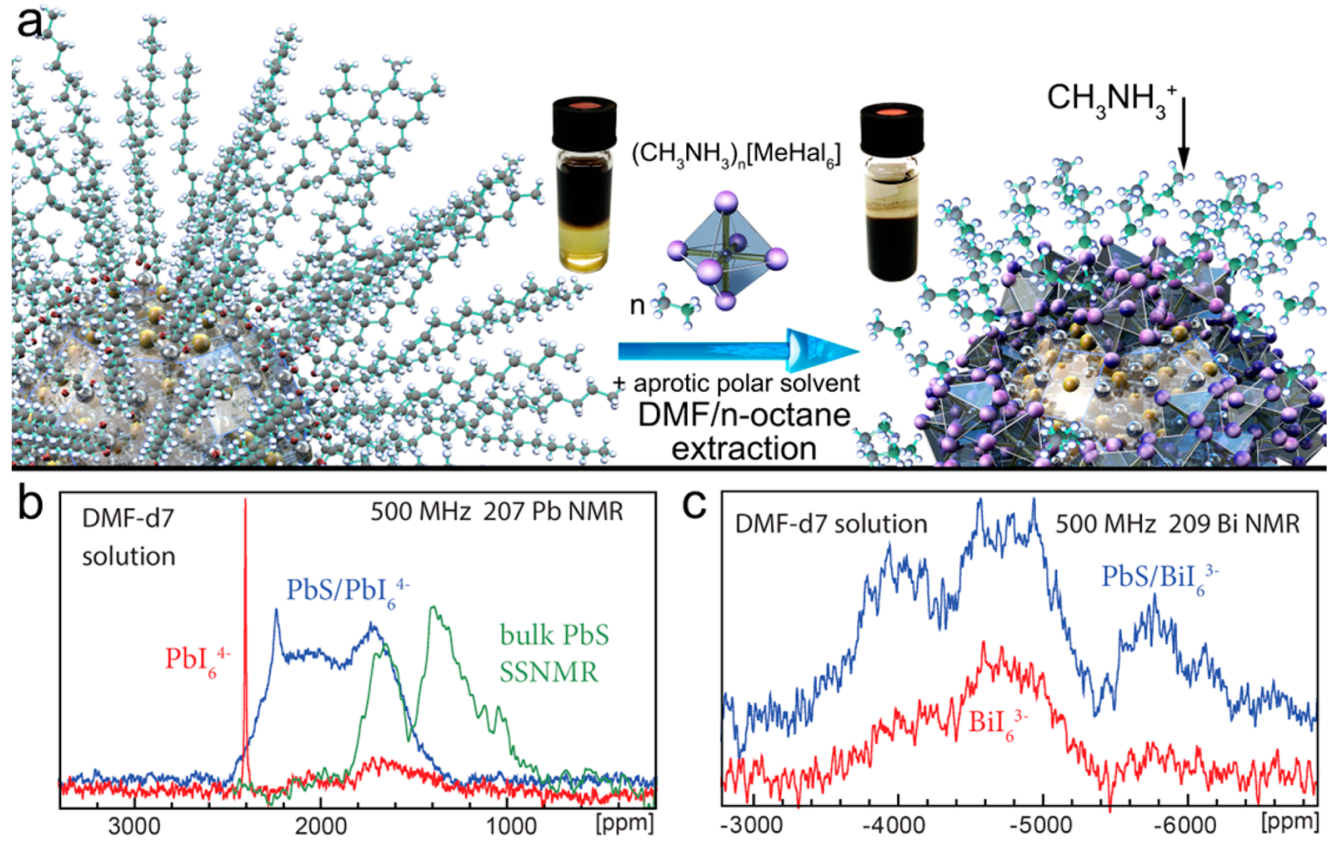

Figure 1. Ligand exchange on PbS nanocrystals. (a) Oleate ligands are replaced by methylammonium metal halides octahedral clusters during a phase transfer from nonpolar to polar solvents. The atoms in the stick and ball models are color coded: $\mathrm{C}$, gray; $\mathrm{H}$, white; halides, violet; metals, silver; $\mathrm{S}$, beige. The vials show the PbS nanocrystals in the nonpolar and polar solvent before and after phase transfer. (b) ${ }^{207} \mathrm{~Pb} \mathrm{NMR}$ spectra of the $\mathrm{PbI}_{6}{ }^{4-}$ clusters in DMF solution, of the $\mathrm{PbS}$ nanocrystals covered by the cluster ligands and solid state NMR spectrum from bulk $\mathrm{PbS}$ for comparison. (c) ${ }^{209} \mathrm{Bi} \mathrm{NMR}$ spectrum of $\mathrm{BiI}_{6}{ }^{3-}$ clusters in DMF and of $\mathrm{PbS}$ nanocrystals covered by these clusters.

Recently, the concept of heteroepitaxial systems based on colloidal nanocrystals has been expanded toward polyheterocrystalline (PHC) solids-colloidal quantum dots embedded in a bulky perovskite matrix, with internally epitaxially aligned heterointerfaces. ${ }^{26}$ The beneficial transport properties of the metal-halide perovskite were merged with the excellent infrared-optical properties of $\mathrm{PbS}$ nanocrystals, for instance, to obtain highly efficient quantum dot near-infrared lightemitting diodes.

Here, we demonstrate $\mathrm{PbS}$ nanocrystals with ligand shells of thin perovskite layers which exhibit an epitaxial relation to the crystals cores. This approach is conceptually different to the previous core/shell nanocrystals, in which an inorganic shell material is applied to optimize surface state passivation while an extra predominantly organic ligand shell is required to maintain colloidal stability. To achieve an epitaxial-ligand shell providing both surface passivation and colloidal stabilization we chose hybrid complex molecules, octahedral metal halide clusters such as $\left(\mathrm{CH}_{3} \mathrm{NH}_{3}\right)_{(6-x)}\left[\mathrm{M}^{(x+)} \mathrm{Hal}_{6}\right]\left(\mathrm{M}^{x+}=\mathrm{Pb}(\mathrm{II}), \mathrm{Bi}(\mathrm{III}), \mathrm{Mn}(\mathrm{II})\right.$, $\mathrm{In}(\mathrm{III}), \mathrm{Hal}=\mathrm{I}, \mathrm{Cl}),{ }^{28-32}$ for the ligand exchange procedure. These were selected because the geometry of the atomic bonds (Figure 1a) is identical to that of the cubic rock-salt structure of the $\mathrm{PbS}$ cores. Furthermore, fitting of the ligand cluster bond lengths to that of the core crystal is shown to provide efficient passivation of the nanocrystal surface, resulting in increased photoluminescence intensity as compared to that of the initial oleate passivated $\mathrm{PbS}$ nanocrystals. The epitaxial ligands are shown to be beneficial for preparation of photoconducting nanocrystal films, exhibiting high on/off ratios of 1000, a linear intensity dependence of the photocurrent, high detectivity of 2 $\times 10^{11} \mathrm{~cm} \mathrm{~Hz}^{1 / 2} / \mathrm{W}$ and a bandwidth of $110 \mathrm{kHz}$. Furthermore, the ligand exchange procedure is so efficient that it can be applied directly after the nanocrystal growth to the crude reaction solution and can be scaled up to get $2.1 \mathrm{~g}$ of the ligandexchanged nanocrystals in a single step. The upscaled ligand transfer enabled the preparation of bulk-samples in the form of wafer pellets, which we used to deduce fundamental electronic parameters of the heteroepitaxial nanocrystal/ligand material. The obtained parameters (carrier mobility of $1.3 \mathrm{~cm}^{2} \mathrm{~V}^{-1} \mathrm{~s}^{-1}$ and carrier lifetime of $69 \mu \mathrm{s}$ ) are promising for device applications, which will benefit from the simple and scalable fabrication process.

\section{RESULTS AND DISCUSSION}

The PbS nanocrystals, for which the quasi-epitaxial ligand shell was developed, were synthesized following a modified procedure of Hines et al. ${ }^{33}$ During and upon synthesis completion the nanocrystals are covered with oleic acid (OA) ligands, allowing dispersion in nonpolar solvents. While the organic ligand shell is appropriate for surface passivation and characterization of the colloidal solution by optical means and electron microscopy, the length of the ligand alkane chain (sketched approximately to scale in Figure 1a) impedes charge transport in nanocrystal films. Thus, a ligand exchange is mandatory for preparation of electronic devices, ${ }^{34,35}$ and many species have been developed for such purposes. ${ }^{36-44}$ Complexes based on precursor solutions of lead halide perovskites have been attempted, such as methylammonium lead iodides and bromides $\left(\mathrm{MAPbI}_{3}, \mathrm{MAPbBr}_{3}\right.$, where $\mathrm{MA}=$ $\left.\mathrm{CH}_{3} \mathrm{NH}_{3}^{+}\right)^{45-48}$ via replacement of OA ligands during a phase transfer procedure from hexane to a polar solvent such as $\mathrm{N}$ methylformamide. This ligand replacement was shown to retain the highly efficient near-infrared photoluminescence of $\mathrm{PbS}$ nanocrystals ${ }^{45}$ and was also applied in solar cells to achieve a record performance for single-step-deposited quantum dot films (power conversion efficiency of $8.95 \%$ ). ${ }^{49}$ Instead of using a standard precursor solution for $\mathrm{MAPbI}_{3}$ perovskites we focused on octahedral clusters of $(\mathrm{MA})_{(6-x)}\left[\mathrm{Me}^{(x+)} \mathrm{Hal}_{6}\right](\mathrm{Me}=$ $\mathrm{Bi}, \mathrm{Pb}, \mathrm{Mn}$, In and $\mathrm{Hal}=\mathrm{I}$ or $\mathrm{Cl})^{28-32}$ in which the cluster 
anion has the same 6-fold coordination as in the rock-salt $\mathrm{PbS}$ crystal (sketched in Figure 1a). These solutions make use of higher (MA)Hal:Me ${ }^{(x+)} \mathrm{Hal}_{x}$ precursor ratios, as compared to $\mathrm{MAMeHal}_{3}$ ligands reported in the literature ${ }^{45-48}$ and thus result predominantly in $\mathrm{MA}_{(6-x)}\left[\mathrm{Me}^{(x+)} \mathrm{Hal}_{6}\right]$ clusters. We focused on $\mathrm{PbI}_{6}^{4-}$ and $\mathrm{BiI}_{6}{ }^{4-}$ clusters, ${ }^{28-30}$ which are obtained in precursor solutions of appropriate stoichiometric compositions. With lower (MA)Hal: $\mathrm{Me}^{(x+)} \mathrm{Hal}_{x}$ ratio solutions in $N, N$ dimethylformamide (DMF), various different coordination molecular geometries are reported, such as tetragonal $\left(\mathrm{Pb}_{2} \mathrm{I}_{6}{ }^{2-}, \mathrm{PbI}_{4}{ }^{2-}\right),{ }^{28,50}$ or pentagonal with squared pyramid shape $\left(\mathrm{PbI}_{3}{ }^{-}\right),{ }^{28,50}$ which do not fit to the crystal structure of the $\mathrm{PbS}$ nanocrystals. Evidences for such multigeometrical molecular arrangements of lead complexes in DMF solution for different ratios of $\mathrm{Pb}^{2+}$ to $\mathrm{I}^{-}$are provided by ${ }^{207} \mathrm{~Pb}$ nuclear magnetic resonance $\left({ }^{207} \mathrm{~Pb} \mathrm{NMR}\right)$ measurements in Figure S1. $\mathrm{MA}_{x} \mathrm{MeHal}_{6}$ clusters ligand replacement is performed via rapid phase transfer of the $\mathrm{PbS}$ nanocrystals from octane to $\mathrm{DMF}$, taking under $10 \mathrm{~s}$ (see methods section). The ligand exchange procedure is as follows: ligand clusters were formed in mixtures of MAI and $\mathrm{PbI}_{2}$ or $\mathrm{BiI}_{3}$ with ratios of $4: 1$ or $3: 1$ in DMF $(6 \%$ mass solution), respectively. An octane solution of nanocrystals $(5 \mathrm{mg} / \mathrm{mL}$, gravimetrically determined) was added and after only one shake of the mixture, the amount of nanocrystals is extracted to the DMF phase. This phase transfer can also be performed from a hexane solution, but the higher boiling point of octane prevents condensation of water onto the cool solvent surface and thus can be carried out under ambient conditions. The successful ligand exchange was apparent due to the obvious phase change of nanocrystals (Figure 1a) and due to the removal of oleic acid (Figure S2). The ligand exchange procedure was found to be so efficient that it can be applied directly to larger amounts of materials, for example, using 100 $\mathrm{mL}$ of crude reaction solution after the nanocrystal growth, where we observed a rapid phase transfer from 1-octadecene solution to DMF phase due to the ligand exchange (Figure S3). In this way, $2.1 \mathrm{~g}$ of $\mathrm{PbS}$ ligand-treated nanocrystals were obtained in a single step. Since the aliphatic solvent and lipophilic byproducts from synthesis are soluble in nonpolar solvents only, a direct ligand exchange can be performed from the crude solution right after nanocrystal growth. Thus, the ligand exchange circumvents tedious washing procedures, which also result in nanocrystal surface degradation.

Formation of the octahedral clusters and their attachment to the nanocrystals was evidenced by a combination of ${ }^{207} \mathrm{~Pb}$ and ${ }^{209} \mathrm{Bi}$ nuclear magnetic resonance (NMR) spectroscopy. To identify the $\mathrm{PbI}_{6}{ }^{4-}$ clusters in DMF solution, external chemical shift references were used (see Experimental methods section), and the spectra in Figure $1 \mathrm{~b}$ are plotted with chemical shifts relative to $\left(\mathrm{CH}_{3}\right)_{4} \mathrm{~Pb}$. The chemical shift of $\mathrm{Pb}\left(\mathrm{NO}_{3}\right)_{2}$ is found at $-2961 \mathrm{ppm}$ and the ${ }^{207} \mathrm{~Pb} \mathrm{NMR}$ resonance of the $\mathrm{PbI}_{6}{ }^{4-}$ ligand clusters is shifted by approximately the same amount $(\delta$ $=-2400 \mathrm{ppm})$, as is expected for its 6-fold coordination (Figure $1 b$, Figure $\mathrm{S} 1$ ). The solid state ${ }^{207} \mathrm{~Pb}$ NMR spectrum of $\mathrm{PbS}$ bulk material, in comparison, exhibited two 200-300 ppm broad peaks at $\sim 1400$ and $\sim 1700 \mathrm{ppm}$. The liquid state ${ }^{207} \mathrm{~Pb}$ NMR spectrum of the nanocrystals, dispersed after ligand exchange in DMF and washed three times to remove excess ligand material, exhibits contributions from both $\mathrm{PbS}$ bulk and the $\mathrm{PbI}_{6}{ }^{4-}$ ligands. The ${ }^{207} \mathrm{~Pb}$ NMR resonances of $\mathrm{PbS}$ are upfield-shifted by about $400 \mathrm{ppm}$ and the sharp NMR resonance of the $\mathrm{PbI}_{6}{ }^{4-}$ ligand exhibited a downfield-shift of $\sim 180 \mathrm{ppm}$ as compared to the $\mathrm{PbI}_{6}{ }^{4-}$ ligand dissolved in DMF, found at $2400 \mathrm{ppm}$. This upfield shift of the $\mathrm{PbI}_{6}{ }^{4-}$ ligand peak evidence the binding of the $\mathrm{PbI}_{6}^{4-}$ clusters to the $\mathrm{PbS}$ nanocrystal surface. Similarly, the spectra of the ${ }^{209} \mathrm{Bi}$ NMR experiments of the $\mathrm{BiI}_{6}{ }^{3-}$ cluster ligands were assigned with the help of $\mathrm{Bi}\left(\mathrm{NO}_{3}\right)_{3}$ as a reference (Figure 1c, Figure S4). After ligand exchange, the ${ }^{209} \mathrm{Bi}$ NMR spectrum of the $\mathrm{PbS}$ nanocrystals also contained broad NMR resonances originating from the $\mathrm{BiI}_{6}{ }^{3-}$ ligand (which are shifted with respect to that of $\mathrm{BiI}_{3}$, Figure S4). In addition, a broad upfield-shifted NMR signal at ca. $-5800 \mathrm{ppm}$ was noted, indicating a possible second type of $\mathrm{BiI}_{x}$ cluster. It should be mentioned that the broadness of the provided bismuth spectra is not a result of other complexes or dynamic exchange, but rather because the ${ }^{209} \mathrm{Bi}$ isotope exhibits in practically all environments broad signals over a wide chemical shift range as it is a quadrupolar nucleus with a spin of $9 / 2$ and a $100 \%$ natural abundance. ${ }^{51}$ The ${ }^{207} \mathrm{~Pb}$ isotope in contrast ( $22.1 \%$ natural abundance) has a nuclear spin of $1 / 2$, which results in sharp and well-defined signals.

An inspection of the nanocrytsals before ligand exchange provides that they are close to spherical in shape and well separated from each other, which is to some extent caused by their organic ligand shell (Figure S5). After ligand exchange their crystalline nature was preserved, the distances between them considerably decreased, and their sizes seemed to be decreased. The size change was quantitatively determined by small-angle X-ray scattering (SAXS) experiments performed in solution (Figure S6a). By fitting the SAXS pattern for OAcapped $\mathrm{PbS}$ in solution to a spherical model, an average core diameter of $3.9 \mathrm{~nm}$ was obtained (note that the light OA ligands essentially do not contribute to the $\mathrm{X}$-rays scattering angle). After ligand exchange to $\mathrm{BiI}_{6}{ }^{3-}$, the average diameter decreased to $3.4 \mathrm{~nm}$. Fitting the $\mathrm{PbS} /\left[\mathrm{BiI}_{6}\right]$ nanocrystals with a spherical core/shell model indicated a shell thickness of around $0.3 \mathrm{~nm}$, corresponding to a $\mathrm{BiI}_{6}{ }^{3-}$ ligand shell thickness of one monolayer. For both data sets a size distribution of about $12 \%$ was found. The overall reduction of nanocrystal radius upon ligand exchange corresponds to $1 / 2$ of the $\mathrm{PbS}$ lattice constant $(0.594 \mathrm{~nm})$, indicating the removal of one monolayer of $[\mathrm{Pb}-$ $\mathrm{S}$ ] atoms during removal of the oleic acid ligands. The SAXS experiments were repeated on thin films of $\mathrm{PbS}$ coated with $\mathrm{OA}$ and $\mathrm{BiI}_{6}{ }^{3-}$ and the dominant peaks in Figure $\mathrm{S} 6 \mathrm{~b}$ correspond to partially ordered, aggregated nanocrystals. Here the oleic acid (OA)-capped nanocrystals show a slightly higher degree of ordering and the derived mean nanocrystal separation of 5.6 $\mathrm{nm}$ is much larger than the pure core diameter of $3.9 \mathrm{~nm}$, clearly indicating nanocrystals separated by OA ligands. On the other hand, the $\mathrm{PbS} /\left[\mathrm{BiI}_{6}\right]$ mean nanocrystal separation was found to be $3.4 \mathrm{~nm}$, matching the total diameter of the core/ ligand-shell NCs. Thus, the nanocrystals with $\mathrm{BiI}_{6}{ }^{3-}$ ligands have direct contact with one another through their inorganic ligand shell.

While the experiments described above confirm successful ligand exchange from $\mathrm{OA}$ to $\mathrm{MA}_{4} \mathrm{PbI}_{6}$ and $\mathrm{MA}_{3} \mathrm{BiI}_{6}$, similar results were obtained with further octahedral metal hexa-halide clusters, such as $\mathrm{MA}_{3} \mathrm{InCl}_{6}$ and $\mathrm{MA}_{4} \mathrm{MnCl}_{6}$. Colloidal solutions in DMF were obtained in all situations with a stability of at least a few days for highly concentrated solutions $(>25 \mathrm{mg} / \mathrm{mL})$. These octahedral cluster ligands performed superiorly in comparison to the previously reported MA metal trihalide ligands ${ }^{45-49}$ in six respects: (i) the ligand exchange reaction is faster (reaction times are below $10 \mathrm{~s}$ in milliliter amounts of solutions); (ii) the resultant colloid is more stable with respect to chemical degradation $\left(\mathrm{BiHal}_{3}\right.$ ligands resulted in $\mathrm{PbS}$ 
nanocrystal solutions which decay into orange-brown precipitates within a day, whereas the hexa-halide capped nanocrystals were found to be completely stable); (iii) the dispersions exhibited improved colloidal stability as a result of considerably higher surface charge, obtained upon treatment with hexahalide ligands-evidenced by zeta-potential measurements on $\mathrm{PbI}_{6}{ }^{4-}$ and $\mathrm{BiI}_{6}{ }^{3-}$ covered nanocrystals (Figure S7), which was found to be more than twice as high as that of $\mathrm{MAPbI}_{3}$ covered nanocrystals from the same batch; (iv) the ability to upscale the ligand exchange reaction by at least 2 order of magnitude (from $15 \mathrm{mg}$ to $2.1 \mathrm{~g}$ ); (v) the ligand exchange reaction can be performed directly after nanocrystal synthesis, avoiding the commonly applied nonsolvent reprecipitation washing procedure which potentially damages the nanocrystals surfaces; (vi) with the octahedral clusters an quasi epitaxial ligand shell can be obtained, as is sketched in the Figure 2a (Figure S8) and is discussed in the following.

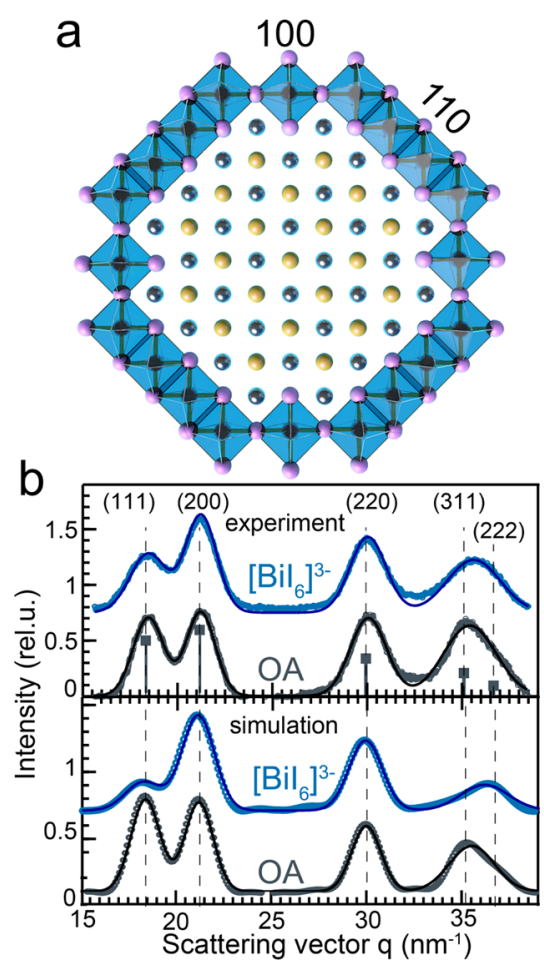

Figure 2. Epitaxial ligand shell. (a) Stick and ball model of a PbS nanocrystal with an epitaxial octahedral methylammonium metal halide cluster shell ([001] projection, color code as in Figure 1). (A model sketch including the methylammonium counterions is shown in Figures S8) (b) Comparison between simulated and measured (symbols) powder X-ray diffraction of oleate (OA) covered and methylammonium bismuth iodide covered $\mathrm{PbS}$ nanocrystals. For the simulation the nanocrystal dimensions are taken as determined by SAXS measurements. The full lines are the fits to the data using Gaussian peaks with background.

An epitaxial interface between a $\mathrm{PbS}$ nanocrystal and a solution processed $\mathrm{MAPbI}_{3}$ perovskite matrix was experimentally demonstrated by Ning et al., ${ }^{26}$ who supported their experimental results with theoretical investigations by calculating a three-dimensional atomistic model of the nanocrystals embedded within the matrix. By reducing the matrix to a thickness of one monolayer an approximate model for the $\mathrm{BiI}_{6}{ }^{3-}$ epitaxial ligand shell was obtained (Figure 2). To form such a shell, the octahedral ligands have to coalesce on the nanocrystal surface, where adhering neighboring clusters share edge- and corner-ions, as could be inferred from the various ligand clusters detected by the Bi-NMR spectra. While the formation of an approximately one monolayer thick $\mathrm{BiI}_{6}{ }^{3-}$ shell was deduced from the rough fits of the SAXS measurements, the epitaxial nature of the shell is suggested by combining the insights from the solid state NMR with those of the high resolution TEM images (Figure S9). The shift observed in the solution NMR of the $\left[\mathrm{PbI}_{6}\right]$ cluster peak, between the ligand solution and the $\mathrm{PbS}$ nanocrystal solution after ligand exchange (Figure 1b, Figure S1) evidenced the attachment of the ligand clusters to the nanocrystals surface. TEM revealed the crystalline nature of the nanocrystals up to the topmost atomic layer, composed of ligands (Figure S9). This interpretation is further supported by wide-angle X-ray scattering (WAXS) measurements that provided a powder X-ray diffraction (XRD) pattern in transmission geometry. The simulated powder pattern of $\mathrm{PbS}$ nanocrystals, with size as deduced from SAX measurements $(3.9 \mathrm{~nm})$, is shown in Figure $2 \mathrm{~b}$ (bottom curve). The pattern, calculated using $\mathrm{PbS}$ bulk lattice parameters, exhibits basically five peaks due to reflexes from differently oriented lattice planes, with various intensities. This simulated spectrum, performed according to the procedure in ref 52 describes the experimental curve for the OA-capped $\mathrm{PbS}$ nanocrystals very well, in terms of peak position and also peak intensity. To simulate the nanocrystals after ligand exchange, the outermost monolayer of $S$ atoms were replaced by $\mathrm{I}$, and the $\mathrm{Pb}$ by $\mathrm{Bi}$, as is suggested by the stick and ball model in the sketch. Furthermore, the total diameter of the nanocrystal was adjusted to $3.4 \mathrm{~nm}$ to take into account the experimentally determined value. Since the lattice structure of the $\mathrm{PbS}$ was simply expanded to the ligand shell, there is essentially no change in the peak positions expected, with respect to OAcapped nanocrystals. The simulation suggests a strong intensity reduction of the (111) peak in comparison to the (200) one which appears to be 2.5 times more intense, as well as a less pronounced reduction of the (311) peak. The experimental data for the $\mathrm{PbS}$ nanocrystals with $\mathrm{BiI}_{6}{ }^{3-}$ clusters are in good agreement with the simulation, performed for a perfect shell with one monolayer thickness. In particular, no peak shifts occurs after ligand exchange because the ligand cation-anion bond length fit to that of the nanocrystals core. The simulated reductions of the (111) and (311) peak intensities are observed in the experimental data (Figure 2b, top curve) as well; however, the decrease in intensity is not as large as expected for spherical nanocrystals with a homogeneous crystalline epitaxial shell. This could be attributed to a slight change of the nanocrystals shape during ligand exchange. Thus, we performed an additional shape retrieval analysis of the SAXS data (see Figure S10), ${ }^{53}$ which reveals a slightly elliptical and faceted mean shape with a slightly larger size distribution for the $\mathrm{PbS}$ / $\mathrm{BiI}_{6}$ core/ligand-shell nanocrystals (for details see Figure S10 and its discussion in the Supporting Information). This nonspherical shape is not only visible in an inhomogeneous electron density distribution as derived from SAXS, but also from a detailed analysis of the XRD spectrum in Figure $2 b$ : a narrower width of the (200) Bragg peak of the $\mathrm{PbS} /\left[\mathrm{BiI}_{6}\right]$ nanocrystals as compared to the other crystal directions suggests nanocrystals with more pronounced (100) facets. These facets might be caused by a preferential shell growth in the [100] direction. Attachment of octahedral $\mathrm{BiI}_{6}{ }^{3-}$ clusters on the $(100) \mathrm{Pb}$ crystal planes therefore should be promoted by matching of the surface (see Figure S11). While the shape 
parameters from SAXS/WAXS are obtained from an ensemble of about $10^{8}$ individual nanocrystals, the nonspherical shape of the $\mathrm{PbS}$ nanocrystals after ligand exchange to $\mathrm{BiI}_{6}{ }^{3-}$ is found by additional TEM investigations of individual $\mathrm{PbS} / \mathrm{BiI}_{6}$ nanocrystals in areas where the nanocrystals are rather densely packed (Figure S12). In such regions several elongated crystalline components extend the idealized spherical shape and form interparticle connections.

An important parameter in heteroepitaxial pseudomorphic growth is the mismatch between the bond lengths in the epitaxial layer and the substrate. Such misfit results in strain, ${ }^{54}$ which is eventually released via formation of dislocation-type defects $^{54}$ at the substrate/epilayer interface or even via formation of three-dimensional islands. ${ }^{55,56}$ Adopting these general statements to the case of nanocrystals covered by a quasi-epitaxial shell, it could be certainly expected that largely different bond length between those in the nanocrystal and from the ligand clusters could heavily strain the nanocrystals cores, cause a highly defected shell or even cause the growth of an incomplete shell. While these cases are hard to prove for 3$4 \mathrm{~nm}$ large nanocrystals directly by electron microscopy, such defected or incomplete shells should have profound effects on the electrical and optical properties of the nanocrystals, and strain imposed on the nanocrystals would result in a changed lattice parameter. To test this hypothesis we applied $\mathrm{BiI}_{6}{ }^{3-}$ and $\mathrm{PbI}_{6}{ }^{4-}$ with closely matching bond lengths to those of the $\mathrm{PbS}$ crystal $(2.97 \mathrm{~A}),^{30,57-62}$ and $\mathrm{InCl}_{6}^{3-}$, and $\mathrm{MnCl}_{6}{ }^{4-}$ with considerably shorter bond lengths. ${ }^{31,32,63}$ The ligand transfer with all four species worked with a yield of $100 \%$ within $10 \mathrm{~s}$ of transfer time with no observable variation in reaction time or colloidal stability of the product. All were applied to the same $\mathrm{PbS}$ nanocrystal batch to obtain comparable results. The ligand exchange to $\mathrm{InCl}_{6}{ }^{3-}$ was again evidenced by XRD spectroscopy (Figure S13) and we observed a clear change in the intensity for [200] and [220] Bragg reflections in comparison to OA-, $\mathrm{MACl}$, or $\mathrm{MA}_{3} \mathrm{BiI}_{6}$-capped nanocrystals (Figure $2 \mathrm{~b}$ ). As discussed above for the case of $\mathrm{MA}_{3} \mathrm{BiI}_{6}$ ligand shells, intensity changes can be attributed to the formation of an inorganic shell and to changes of the nanocrystal shape. Furthermore, these peaks were seen to be slightly shifted with respect to the corresponding peaks measured for the OA-capped $\mathrm{PbS}$, pointing to strain imposed by the ligand shell on the nanocrystals. Although we did not closely examine these spectra, these results can be quoted as direct evidence for the successful ligand exchange. We tested photoluminescence of resultant nanocrystals suspended in DMF and photoconductivity of nanocrystal films deposited on interdigitate electrodes in lateral orientation. A photoluminescence enhancement is observed upon ligand exchange from $\mathrm{OA}$ to $\mathrm{PbI}_{6}{ }^{4-}$ clusters (Figure 3a). This enhancement evidences the high quality of the obtained ligand shell, as well as a type I straddling band alignment between the core and ligand shell. In the methylammonium perovskite system, the chlorides generally exhibit a larger band gap than the iodides. ${ }^{64}$ Therefore, the $\mathrm{InCl}_{6}{ }^{3-}$ and $\mathrm{MnCl}_{6}{ }^{4-}$ clusters used to form the ligand shell should also preserve the nanocrystals luminescence. Indeed luminescence was observed; however, its intensity decreased by more than a factor of 20 . Thus, it can be assumed that $\mathrm{InCl}_{6}{ }^{4-}$ and $\mathrm{MnCl}_{6}{ }^{4-}$ clusters form a defected shell due to the mismatch between the bond lengths of the ligand clusters and that of the $\mathrm{PbS}$ nanocrystals. In this respect it should be noted that the spectral shape of the photoluminescence is affected by strong absorption lines of the DMF solvent (Figure S14). Thus, size-
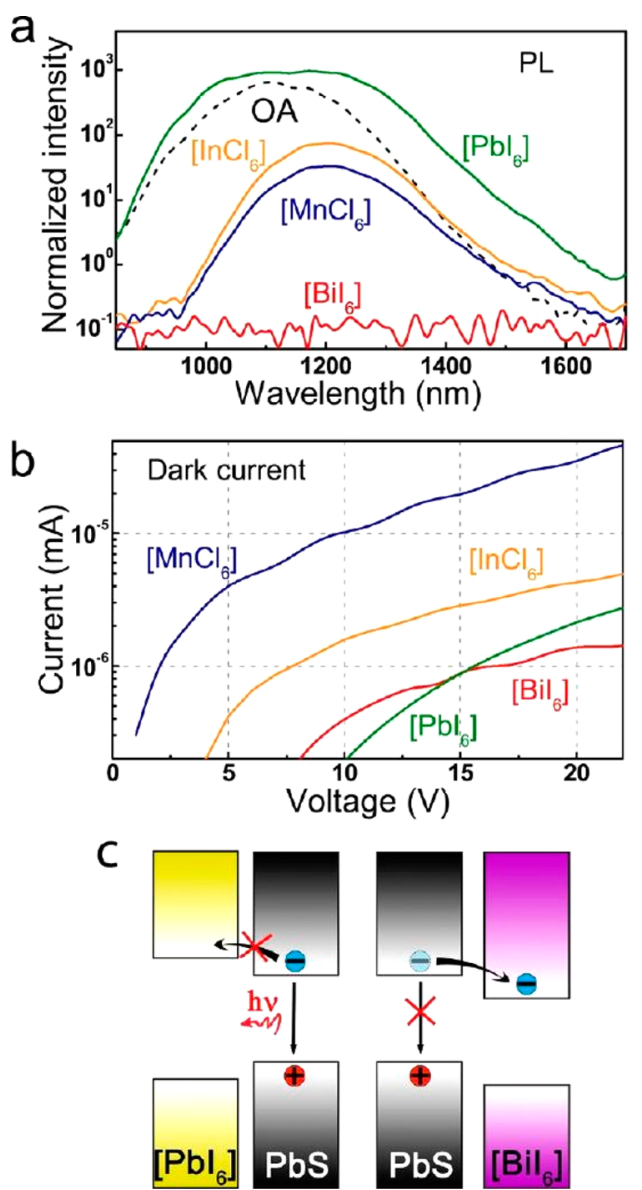

Figure 3. Ligand shell properties. (a) Photoluminescence of equally concentrated $\mathrm{PbS}$ nanocrystals solutions with different ligand species. (b) Dark currents measured in films of PbS nanocrystals, covered with octahedral clusters of methylammonium metal halides with different bond length. (c) Type of band alignment between $\mathrm{PbS}$ core and $\mathrm{MA}_{x} \mathrm{MeHal}_{6}$ ligand shell, as suggested by the photoluminescence experiments.

related peak shifts could not be observed accurately and superimposed absorption lines may effect spectral shape. Nevertheless, we find a clear relation between the PL intensity and $\Delta L=a_{\mathrm{PbS}}-a_{\left[\mathrm{MeHal}_{6}\right]}$, the difference in bond lengths between the nanocrystal and ligand cluster, and the corresponding lattice mismatch $(\%)\left(\Delta L / a_{\mathrm{PbS}}\right) \times 100$ (Figure $\mathrm{S} 15$, the mismatches of the metal halide clusters used are $-3.8 \%$ to $1.8 \%$ for $\left[\mathrm{BiI}_{6}\right],{ }^{28,30,58}-20 \%$ to $0.3 \%$ for $\left[\mathrm{PbI}_{6}\right]^{28,29,50,59-62,64} 12.4 \%$ to $19 \%$ for $\left.\left[\mathrm{InCl}_{6}\right]\right]^{32,63} 23 \%$ to $24.6 \%$ for $\left.\left[\mathrm{MnCl}_{6}\right]^{31}\right)$. There is an approximately exponential decay of the luminescence intensity with respect to $\Delta L$ and lattice mismatch. In Figures S15-S17 error bars are added to the values of the metal halide bond length, included to account for the range of $\mathrm{Me}-\mathrm{Hal}$ bond lengths reported for hexahalide clusters in the literature. ${ }^{28-32,50,58-64}$ The variation of reported bond lengths is generated by diverse environments for the clusters, in particular by different counterions applied. The bond lengths are stretched or squeezed to fit a geometry which minimizes the cluster's Gibbs Free Energy, which varies for different counterions. Disregarding such variation, transport results were found to fall in line with photoluminescence quenching, where the bond-length-mismatched chloride ligands provide substantially higher dark currents as compared to the $\mathrm{PbI}_{6}^{4-}$ (Figure 3b, Figure S16). Obviously, defected or 
incomplete shells impede charge transport between nanocrystals much less than perfect shells; perfect shells provide energy barriers toward charge transport due to a straddling band alignment between the nanocrystal cores and their ligand shells.

With respect to the optical and electronic properties, the nanocrystals covered by $\mathrm{BiI}_{6}{ }^{3-}$ clusters, whose bond lengths show a similarly good lattice matching to $\mathrm{PbS}$ as the $\mathrm{PbI}_{6}{ }^{4-}$, represent an interesting exceptional case. These nanocrystals provide in films a similarly low dark current as the $\left[\mathrm{PbI}_{6}\right]$ covered ones, but in solution a complete luminescence quenching is observed. The quenching points to a staggered type II band alignment between core and ligand shell, causing rapid exciton separation and capture of one carrier type, thus rendering it unavailable for the geminate pair emission responsible for nanocrystal luminescence (as is sketched in Figure 3c). Thus, the situation is comparable to the case of IIVI semiconductors, for which photoluminescence quantum yields are also strongly related to surface states, provided by the ligand attachment. In the case of $\mathrm{CdSe}$, as an example, amines used as ligands provide bright luminescence, whereas thiols form states within the band gap, ${ }^{65}$ resulting in a luminescence quenching. Similarly, in $\mathrm{PbS}$ nanocrystals oleic acid capping results in strong luminescence, whereas a ligand exchange to electron accepting methanofullerene carboxylate based ligands resulted in strongly quenched photoluminescence via rapid exciton dissociation, which was proven to be beneficial for the photoconductivity in nanocrystal films. ${ }^{66}$

Lattice matching and band alignments are also highly relevant in thin film devices, which we used to demonstrate the potential of the inorganically capped nanocrystals for infrared photodetection. First, it should be noted that with all tested ligands photoconductivity was obtained under white light illumination with a power density of $80 \mathrm{~mW} / \mathrm{cm}^{2}$, at which a clear increase of current was observed. The light on/off ratios are shown in the inset of Figure 4a to vary between values of $\sim 15$ and 1000 . Here again the performance scales with lattice mismatch, as is shown in more detail in Figure S17. The smallest on/off ratio was provided by $\mathrm{MnCl}_{6}{ }^{4-}$ covered nanocrystals and the highest by those covered with $\mathrm{BiI}_{6}{ }^{3-}$. The $\mathrm{PbS}$ nanocrystal cores are embedded within ligand films of metal-organic perovskite semiconductors, known to provide high photocurrents themselves; ${ }^{67-69}$ however, the measured photoconductivity spectra clearly exhibit the characteristic spectral features of PbS nanocrystals (Figure 4a). Passivated by $\mathrm{OA}$ and suspended in toluene, the solution excitonic absorption peak was observed close to $1050 \mathrm{~nm}$, whereas in a thin film the $\mathrm{MA}_{3} \mathrm{PbI}_{6}$ - and $\mathrm{MA}_{3} \mathrm{BiI}_{6}$-capped nanocrystals displayed an excitonic absorption feature shifted toward the infrared by $\sim 80 \mathrm{~nm}$ (Figure $4 \mathrm{a}$ ). Red shifts between solution and film are typically attributed to electronic coupling between the nanocrystals, or to an alteration of the nanocrystals' environmental dielectric constant. ${ }^{70,71}$ Both effects certainly contribute to the responsivity spectra in the present case; however, no accurate value for the shift upon film formation can be provided. After ligand exchange the nanocrystals are solved in DMF or similar polar solvents, exhibiting numerous molecular absorption bands in the near IR regime (Figure S14), disabling measurement of any meaningful nanocrystal absorption spectra in solution. Importantly, the photoconductivity spectrum closely follows the absorbance measured in the films (Figure S18). At $1150 \mathrm{~nm}$ the responsivity reaches $1.4 \mathrm{~A} / \mathrm{W}$ (Figure 4a) for both the $\mathrm{BiI}_{6}{ }^{3-}$ - and the $\mathrm{PbI}_{6}{ }^{4-}$-capped nanocrystals (at a modulation frequency of $10 \mathrm{~Hz}$ and detector
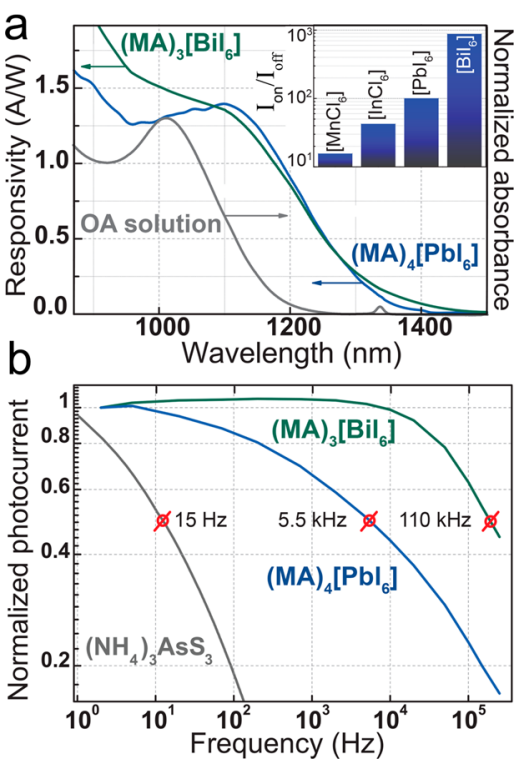

Figure 4. Photoconductor characteristics. (a) Responsivity spectra of $\mathrm{PbS}$ nanocrystals with octahedral $\mathrm{BiI}_{6}{ }^{3-}$ and $\mathrm{PbI}_{6}{ }^{4-}$ ligand cluster shells, compared to the absorption spectrum obtained with an oleate (OA) shell. The inset shows the current ratios measured under white light illumination and in darkness. (b) Responsivity value taken at the wavelength of the excitonic absorption feature as a function of modulation frequency. For comparison, the frequency dependence of a film prepared from $\left(\mathrm{NH}_{4}\right)_{3} \mathrm{AsS}_{3}$-covered $\mathrm{PbS}$ nanocrystals, measured under identical conditions, is shown (ref 73).

area of $0.1 \mathrm{~cm}^{2}$ ) which provides, with the measured noise current of $70 \mathrm{fA} / \mathrm{Hz}^{1 / 2}$, a specific detectivity of $2 \times 10^{11} \mathrm{~cm}$ $\mathrm{Hz}^{1 / 2} \mathrm{~W}^{-1}$. It should be noted that the noise current represents an upper limit, because it includes contributions from the setup which are not subtracted from the measured value, so as to provide a lower limit for the detectivity. While the detectivity was deduced for an illumination power of $1 \mathrm{nW}$, the responsivity was found to be almost intensity independent by measuring a linear dependence of the photocurrent on illumination power (Figure S19). The presently obtained responsivity values correspond closely to a product of external quantum efficiency times a photoconducting gain of $100 \%$. For the nanocrystal photoconductors illuminated by an infrared light emitting diode at the wavelength of the excitonic peak, the frequency dependence of the $\mathrm{PbI}_{6}{ }^{3-}$ nanocrystal film provided a continuously decreasing photocurrent and the $-3 \mathrm{~dB}$ cutoff frequency was $5.5 \mathrm{kHz}$ (Figure $4 \mathrm{~b}$ ). In contrast, $\mathrm{BiI}_{6}{ }^{3-}$ capping provided no photocurrent decrease up to $>10 \mathrm{kHz}$ and the measured cutoff frequency of $110 \mathrm{kHz}$ was limited by the lockin amplifier. Thus, the frequency dependence delivers an upper limit for the time response of $9 \mu \mathrm{s}$. These results, time response and detectivity of the $\mathrm{BiI}_{6}{ }^{3-}$ covered nanocrystal films, can be directly compared to those obtained recently with arsenicsulfide-encapsulated $\mathrm{PbS}$ nanocrystal films, measured in the same configuration. For $\left(\mathrm{NH}_{4}\right)_{3} \mathrm{AsS}_{3}$-capped $\mathrm{PbS}$ nanocrystals the photoresponse exhibited a cutoff frequency of $15 \mathrm{~Hz}$, due to massive carrier trapping, which resulted in a high photoconductive gain, responsivity, and detectivity $\left(1.2 \times 10^{13}\right.$ Jones). Thus, the product of detectivity times bandwidth gives a value of $1.8 \times 10^{14} \mathrm{~cm} \mathrm{~Hz}^{3 / 2} / \mathrm{W}$, whereas in the $\mathrm{BiI}_{6}{ }^{3-}$ covered nanocrystal films a superior value of $2.2 \times 10^{16} \mathrm{~cm}$ $\mathrm{Hz}^{3 / 2} / \mathrm{W}$ is obtained. This higher product results from the 
favorable band alignment (Figure 3c), suggesting separate channels for the electron and hole conduction. Electrons are conducted through the $\mathrm{BiI}_{6}{ }^{3-}$ perovskite ligand material, which form during attachment of the shells and soft annealing a conducting network around the nanocrystals. The holes are conducted in the valence band states of the $\mathrm{PbS}$ nanocrystal cores and eventually tunnel through the monolayer-thin $\mathrm{BiI}_{6}{ }^{3-}$ shells. The assumed type II band alignment between the PbS core and a conducting $\mathrm{BiI}_{6}{ }^{3-}$ ligand network in the film enables absorption of photons with energies below the band gap of the $\mathrm{PbS}$ nanocrystals, namely from valence band states of the $\mathrm{PbS}$ cores to the conduction band states of the $\mathrm{BiI}_{6}{ }^{3-}$ shell. Indeed such core-shell transitions are observed in the photoconductivity spectra of $\mathrm{PbS} / \mathrm{BiI}_{6}$ core/ligand shell nanocrystal films, by a weak sub-band gap tail reaching up to $1500 \mathrm{~nm}$ wavelength (Figures S18 and S20). This is in contrast to the spectra of the samples with $\mathrm{PbI}_{6}^{4-}$ ligands, for which this tail is missing, due to the type I band alignment, between core and shell materials. Thus, the superior photoconducting behavior of the $\mathrm{BiI}_{6}{ }^{3-}$-covered $\mathrm{PbS}$ nanocrystals, compared to other octahedral clusters, results not only from superior matching of ligand bond length to the $\mathrm{PbS}$ core, but also from appropriate electronic band alignment. The latter depends on nanocrystal size and predicts a lower photoconductivity behavior, but improved luminescence for larger $\mathrm{PbS}$ nanocrystals. This is in agreement with experiment: for OA-capped $\mathrm{PbS}$ nanocrystals with an emission peak at $1120 \mathrm{~nm}, \mathrm{BiI}_{6}{ }^{3-}$ ligands resulted in luminescence quenching by at least a factor of 84 000. For larger nanocrystals, with an emission wavelength peak at $1500 \mathrm{~nm}$ after ligand exchange, a smaller quench ratio of 4000 is observed (Figure S21), pointing to a closer energy matching of the nanocrystals core states and its ligand states. The responsivity spectra of the $\mathrm{PbS}$ nanocrystal films deliver further evidence for the details of the ligand exchange and the importance of the lattice matching: in the case of $\mathrm{MA}_{3} \mathrm{InCl}_{6}$ or $\mathrm{MA}_{3} \mathrm{MnCl}_{6}$ ligands the photoresponsivity spectra exhibit signals up to substantially longer wavelengths as for the $\mathrm{MA}_{3} \mathrm{PbI}_{6}$ - and $\mathrm{MA}_{3} \mathrm{BiI}_{6}$-capped nanocrystals (Figures S22) without any excitonic features. This red-shifted cutoff wavelength points to a larger nanocrystal size after ligand exchange, which is in agreement with the red-shifted PL spectra reported in Figures $3 a$, and/or to the presence of sub-bandgap states. Such subbandgap states can be attributed to defects, which can be expected to be formed in an epitaxial layer with a large lattice mismatch in respect to the substrate. Thus, the lattice matching between the ligand clusters and the nanocrystal core material heavily influences the luminescence quantum yield in solution, the dark currents, responsivity, and detectivity in nanocrystal films, and the shape of the spectral response in solutions and in films. Defect-free ligand shells are obtained with lattice matching ligand clusters, which leads to superior optical and electronic properties.

To evaluate the electronic properties of $\mathrm{MA}_{3} \mathrm{BiI}_{6}$ ligandcapped $\mathrm{PbS}$ nanocrystal solids quantitatively, we have made use of the upscaled ligand exchange procedure to provide gram scale amounts of materials, required to process bulk samples. In particular, with a pellet press, wafer samples with thicknesses between 0.3 and $0.6 \mathrm{~mm}$, a diameter of $13 \mathrm{~mm}$ (Figures 5a), and a density which was $66 \%$ of that of bulk $\mathrm{PbS}$, were formed. After sputtering $\mathrm{Pt}$ contacts on both sides of the pellets, photoresponsivity spectra were measured to prove that the quantum confinement is retained even after applying a pressure of $190 \mathrm{MPa}$ to the powdered material. The photoconductivity
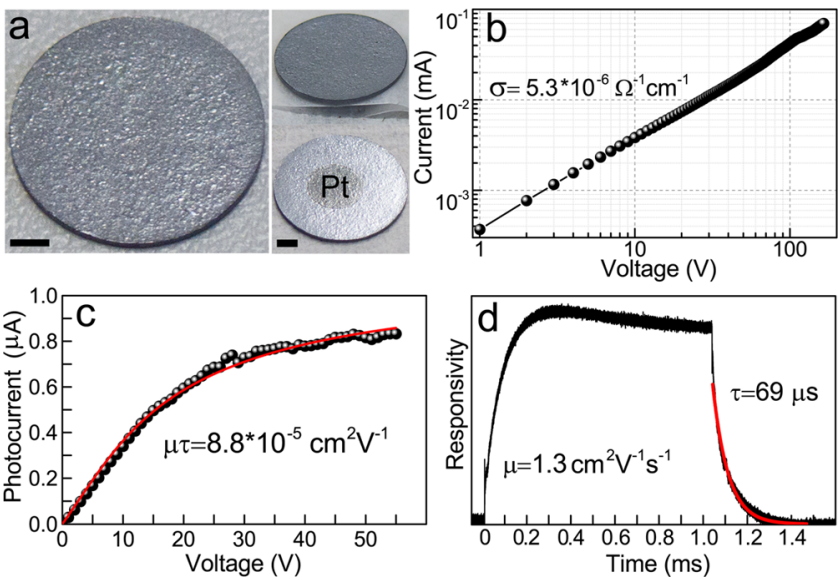

Figure 5. Properties of $\mathrm{MA}_{3} \mathrm{BiI}_{6}$ ligand-capped $\mathrm{PbS}$ nanocrystal solids. (a) Photos of the wafer-shaped samples with a diameter of $13 \mathrm{~mm}$ (scale bare is $2 \mathrm{~mm}$ ), bare, and with a sputtered Pt contact on top of it. (b) $I-V$ characteristics measured in quasi-steady-state conditions. (c) Voltage dependence of the photocurrent and fit of the Hecht equation providing the given mobility lifetime product. (d) Transient photoconductivity response to a $1 \mathrm{~ms}$ light pulse, showing an exponential decay with a fitted lifetime of $69 \mu \mathrm{s}$.

spectra were measured in vertical direction through the sample and a cutoff was clear at a wavelength of $\sim 1450 \mathrm{~nm}$ (Figures S23), far shorter than the band gap of bulk PbS. The shape of the photoconductivity spectrum is different from that of the thin films, because the larger sample length favors photoconductivity signals from weakly absorbing spectral regions with sufficiently higher penetration lengths, than that above the materials bandgap energy. In the present pellets, the region of strong absorbance reaches up to $1450 \mathrm{~nm}$ (Figures S23). At shorter wavelengths the sample is completely opaque, whereas at longer wavelengths, in between 1520 and $1670 \mathrm{~nm}$, a strong absorbance due to the $\mathrm{CH}_{3} \mathrm{NH}_{3}$ vibrations is found, eventually also affecting the shape of the pellets photoresponsivity spectrum. $I-V$ characterization was applied to obtain the conductivity of the sample $\left.\left(\sigma=5.3 \times 10^{-6} 1 / \Omega \mathrm{cm}\right)\right)$. Biasing the sample mounted on a heat sink in quasi continuous mode up to $165 \mathrm{~V}$ resulted in a perfectly linear $I-V$ curve (Figure $5 \mathrm{~b}$ ), with no nonlinearities at higher biases indicative for carrier trapping. With the same pulsed quasi-continuous mode biasing the field dependence of the photoconductivity was measured, which can be fitted by the Hecht formula ${ }^{72}$ to provide a mobility lifetime product of $\mu \tau=8.8 \times 10^{-5} \mathrm{~cm}^{2} / \mathrm{V}$ (Figure $5 \mathrm{c}$ ). To obtain the carrier lifetime, transient photoconductivity was measured from the pellets, and from the photoconductivity decays a lifetime of $69 \mu \mathrm{s}$ is deduced (Figure 5d). This lifetime is somewhat higher than the lifetime deduced from the frequency-dependent experiments shown in Figure $4 b$, however the sample preparation and geometry is completely different. With this lifetime, a carrier mobility of $1.2 \mathrm{~cm}^{2} /(\mathrm{V} \mathrm{s})$ is deduced. Using the hot probe technique, the $\mathrm{PbS}$ nanocrystal pellets were found to be n-type and with the measured mobility the electron concentration was determined to be $2.6 \times 10^{13}$ $\mathrm{cm}^{-3}$. Such a small residual carrier concentration, which corresponds to about $10^{-6}$ carriers per nanocrystal, points to a low impurity or defect concentration in the nanocrystal bulk material, which is certainly beneficial for applications of this type of heteroepitaxial nanocrystal semiconductor, prepared by an upscaled ligand exchange method. 


\section{CONCLUSIONS}

Epitaxial growth techniques which are of paramount importance for electronic device fabrication have been expanded here via a facile ligand exchange, performed on colloidal $\mathrm{PbS}$ nanocrystals. The epitaxial nature of the ligand shell was proven by (i) a luminescence increase in solution after ligand exchange, (ii) the strong dependence of the optical and electrical properties on lattice mismatch, (iii) by agreement of powder XRD spectra to simulated results assuming an epitaxial shell, and (iv) by knowing from solution NMR that the ligand clusters bind to the nanocrystals, which after ligand exchange show lattice fringes in high resolution TEM images until the outermost atomic layer. For $\mathrm{PbS}$ nanocrystals the $\mathrm{MA}_{3} \mathrm{BiI}_{6}$ ligands are ideally suited to provide a coherent core/ligand shell interface with alignment of energy states to provide charge separation. The performance of photoconducting films with a high detectivity-times-bandwidth product and the parameters deduced from bulk samples are promising for future device developments. The strategy to attach zero-dimensional clusters to nanocrystals providing epitaxial matching ligand shells has the potential to be expanded to further nanocrystal core materials. Such a development could trigger substantial improvements in all forms of nanocrystal-based electronic devices that suffer from carrier trapping, interfacial carrierrecombination, or high impedances.

\section{EXPERIMENTAL SECTION}

Materials. Chemicals. Methylamine solution 33 wt \% in absolute ethanol, hydriodic acid 57 wt \% in water (contains no stabilizer, 99.99\% trace metals basis), anhydrous diethyl ether were received from Sigma-Aldrich. Lead(II) iodide (99.9985\%), bismuth(III) iodide (99.995\%), anhydrous toluene, and anhydrous $\mathrm{N}, \mathrm{N}$-dimethylformamide (DMF) were bought from Alfa Aesar. Absolute ethanol was supplied by Merck. All chemicals were used as received.

PbS Nanocrystals Synthesis. A $3.9 \mathrm{~nm}$ sample of PbS NCs was synthesized with the help of a modified method by Hines et al. ${ }^{33} \mathrm{~A}$ $17.2 \mathrm{~mL}$ aliquot of octadecene (ODE), $0.45 \mathrm{~g}$ of $\mathrm{PbO}$, and $2.8 \mathrm{~mL}$ of oleic acid $(\mathrm{OA})$ were loaded into a $50 \mathrm{~mL}$ three-necked flask. The reagents were dried under vacuum at $150{ }^{\circ} \mathrm{C}$ for $90 \mathrm{~min}$, and then kept at $150{ }^{\circ} \mathrm{C}$ and under argon flow for another $30 \mathrm{~min}$. During this time lead(II) oleate was formed in situ, indicated by the loss of color in the reaction mixture. Then, $0.21 \mathrm{~mL}$ of hexamethyldisilathiane (HMDT) was dissolved in $10 \mathrm{~mL}$ of dried ODE in a glovebox, and this mixture was swiftly injected into the reaction mixture at $150{ }^{\circ} \mathrm{C}$. The heating mantle was removed after HMDT injection to allow cooling of the three-neck flask to $100{ }^{\circ} \mathrm{C}$ then reinstalled to keep the flask at $100{ }^{\circ} \mathrm{C}$ for approximately $5 \mathrm{~min}$. At this point the reaction was quenched by rapidly cooling the solution in a water bath. PbS NCs were washed via dispersion in hexane and ethanol followed by centrifugation. The washing was repeated three times and, upon completion, all solid residues were dissolved in $4 \mathrm{~mL}$ of water-free toluene and stored inside a glovebox. The final nanocrystal size can by controlled by changing the ratio between 1-octadecene and oleic acid.

$\mathrm{CH}_{3} \mathrm{NH}_{3}$ l. Synthesis of $\mathrm{CH}_{3} \mathrm{NH}_{3} \mathrm{I}$ took place by a simple neutralization reaction of methylamine with hydroiodic acid: $20 \mathrm{~mL}$ of 57 wt \% hydroiodic acid was slowly added dropwise to a cooled flask of $100 \mathrm{~mL}$ of absolute ethanol and $35 \mathrm{~mL}$ of $33 \mathrm{wt} \%$ methylamine with vigorous stirring. After half an hour the solvent was evaporated resulting in formation of a brown crystalline powder. This was washed twice with $100 \mathrm{~mL}$ of anhydrous diethyl ether by stirring for $30 \mathrm{~min}$ and decanting the solvent. The powder was then refluxed with $100 \mathrm{~mL}$ of ethyl ether for $2 \mathrm{~h}$ followed by cooling to $5{ }^{\circ} \mathrm{C}$ and decanting the liquid phase. For the final purification step, the powder was recrystallized from anhydrous ethanol/toluene 5:1 solution and crystals were collected on a Schott funnel via vacuum filtering. The final white crystals were dried under vacuum for $24 \mathrm{~h}$ and stored inside a nitrogen glovebox.

$\mathrm{MA}_{4} \mathrm{Pbl}_{6}$ and $\mathrm{MA}_{3} \mathrm{Bil}_{6}$. were prepared by mixing oMAI with $\mathrm{PbI}_{2}$ or $\mathrm{BiI}_{3}$ at a 4:1 and 3:1 molar ratio respectively together with anhydrous DMF inside the glovebox.

Ligand Exchange. For the ligand exchange, $2.4 \mathrm{~mL}$ of DMF containing a $6.5 \mathrm{wt} \%$ mixture of either bismuth or lead perovskite complex was added to $4 \mathrm{~mL}$ of $5 \mathrm{mg} / \mathrm{mL} \mathrm{PbS} \mathrm{NCs} \mathrm{hexane} \mathrm{solution}$ and the mixture was shaken for $10 \mathrm{~s}$. During this time the complete extraction of black nanocrystals from the upper hexane layer to the lower DMF solution occurred. The upper hexane layer was removed using a pipet, and a further $4 \mathrm{~mL}$ of anhydrous hexane was added. The mixture was shaken again for $15 \mathrm{~s}$ and then the hexane was discarded. This anhydrous hexane rinsing was performed twice more followed by the addition of $3.2 \mathrm{~mL}$ of anhydrous toluene and centrifugation at $6000 \mathrm{rpm}$ for $3 \mathrm{~min}$. The supernatant was discarded, and $1 \mathrm{~mL}$ of anhydrous DMF was used to dissolve black solid residuals to prepare for the next reprecipitation by adding of $3.2 \mathrm{~mL}$ of anhydrous toluene and centrifugation at $6000 \mathrm{rpm}$ for $3 \mathrm{~min}$. The final solid was dissolved in $0.65 \mathrm{~mL}$ of DMF and filtered through a $0.2 \mathrm{~mm}$ Nylon syringe filter. The obtained solution was used immediately for film fabrication.

Upscaled Ligand Exchange of PbS/[Bii $\left.{ }_{6}\right]$ Nanocrystals. The synthesis of $\mathrm{PbS}$ nanocrystals described above was first scaled up by a factor of 5. After the crude solution was cooled to room temperature, the reaction mixture was poured into a separation funnel containing a filtered (0.45 mm PTFE) mixture of $85 \mathrm{~mL}$ of DMF, $11.18 \mathrm{~g}$ of $\mathrm{BiI}_{3}$, and $9.04 \mathrm{~g}$ of MAI. The funnel was vigorously shaken for $10 \mathrm{~s}$, and subsequently the complete phase separation took place within $5 \mathrm{~min}$. The black DMF portion was released into a second separation funnel, and $100 \mathrm{~mL}$ of octane was added to repeat the phase separation procedure. This octane rinsing step was repeated two more times. After the final rinsing, the DMF solution from the bottom of the funnel was poured to $3 \times 50 \mathrm{~mL}$ centrifuge tubes, to which $5 \mathrm{~mL}$ of toluene was added, before the mixture was centrifuged at $6000 \mathrm{rpm}$ for $5 \mathrm{~min}$. The supernatant was decanted and a black solid precipitate was dispersed in $15 \mathrm{~mL}$ of DMF. The dispersion was filtered through 0.45 $\mu \mathrm{m}$ PTFE, and another precipitation step was done with $9 \mathrm{~mL}$ of toluene. After centrifugation for a second time, the solid residual was redispersed in DMF, or used as an ink for device preparation, or dried and used for pellet pressing. The same procedure can be used for another octahedral metal halides cluster ligand exchange, but with corrections of precursor masses according to molar amounts.

Pellet Preparation. A controllable pressure pellet press and toolkit with $13 \mathrm{~mm}$ of inner diameter were used to prepare the pellets. Prior to pressing, the powder was dried for $2 \mathrm{~h}$ inside the nitrogen glovebox at $80{ }^{\circ} \mathrm{C}$. A pressure of $10 \mathrm{t}$ was applied for $5 \mathrm{~min}$ to $200-400 \mathrm{mg}$ batches of ground $\mathrm{PbS} /\left[\mathrm{BiI}_{6}\right]$ powder.

Substrate Preparation for Thin Films. Gold contacts were prepared on glass substrates by optical lithography and thermal evaporation followed by a lift-off process. These electrodes are films of $\mathrm{Au} / \mathrm{Ti}$ with $40 / 10 \mathrm{~nm}$ thickness. The size of the gap between electrodes was 10 $\mu \mathrm{m}$ and total length was $8 \mathrm{~mm}$ giving an $8 \times 10^{-8} \mathrm{~m}^{2}$ active area, except for the noise characterization, which was done with larger active areas of $0.1 \mathrm{~cm}^{2}$.

Film Fabrication. Films were fabricated by drop-casting PbS NCs in DMF solution onto prepatterned finger-structured gold contacts. The gold contacts were then dried on a heating plate at $80^{\circ} \mathrm{C}$ for $2 \mathrm{~h}$ inside the glovebox.

Photoconductivity. Photoconductivity (PC) was performed by illumination with a tungsten lamp monochromatized through an Acton SP2150 (Princeton Instruments) spectrograph/monochromator. PC spectra were recorded for light modulated by a mechanical chopper within a frequency of $33 \mathrm{~Hz}$. Bias was applied by a Keithley $236 \mathrm{SMU}$ at $10 \mathrm{~V}$, chosen according to the stability of the dark current. The signal was recorded by a Signal Recovery 7265 DSP lock-in amplifier, which provides as a standard output also the noise voltage or current per bandwidth. The setup was controlled by home-written software in LabView IV. Characteristics were measured by the Keithley 236 SMU with and without illumination of a tungsten lamp of intensity $20 \mathrm{~mW}$ $\mathrm{cm}^{-2}$. Frequency dependencies were recorded with a load resistance of 
$50 \mathrm{Ohm}$ and pulsed (square shape) focused illumination from a LED1070E (Thorlabs) emitting light with a central wavelength of $1070 \mathrm{~nm}$.

$I-V$. Measurements for the pressed pellet were performed using a Keithley 2612B source meter controlled by a home-written software in Python under light and dark conditions between 0 and $165 \mathrm{~V}$. Quasicontinuous state operation was achieved by mounting the pellet to a heat sink with conducting silver paste, and by measuring the current with $0.2 \mathrm{~s}$ long voltage pulses, which were sufficiently separated to allow complete sample cooling (300 s).

Solution and Solid State Nuclear Magnetic Resonance (NMR). Measurements were taken on a Bruker Avance III $500 \mathrm{MHz}$ spectrometer equipped with a cross-polarization (CP) magic-angle spinning (MAS) probe. ${ }^{13} \mathrm{C} \mathrm{CP-MAS} \mathrm{spectra} \mathrm{were} \mathrm{recorded} \mathrm{at} 125$ $\mathrm{MHz}$ at a spinning rate of $10 \mathrm{kHz}$ at a temperature of $298 \mathrm{~K}$. Measurements at a spinning rate of $10 \mathrm{kHz}$ and ${ }^{13} \mathrm{C}$ CP-TOSS MAS were recorded, and spectra were compared to identify side bands. ${ }^{13} \mathrm{C}$ attached proton test (APT) NMR spectra were recorded on a Bruker Avance III $700 \mathrm{MHz}$ spectrometer with pulse sequences provided by the manufacturer, and standard deuterated solvents were used as internal calibration standards.

Solution and solid state ${ }^{207} \mathrm{~Pb}$ NMR and ${ }^{209} \mathrm{Bi}$ NMR experiments were recorded at 104.6 and $80.4 \mathrm{MHz}$, respectively, at a temperature of $298 \mathrm{~K}$. In solution, $\mathrm{Pb}\left(\mathrm{NO}_{3}\right)_{2}(1.0 \mathrm{M})$ in $\mathrm{D}_{2} \mathrm{O}(\mathrm{pH} 3.3)$ is used as an external chemical shift reference for ${ }^{207} \mathrm{~Pb}$ because of its lower toxicity than $\left(\mathrm{CH}_{3}\right)_{4} \mathrm{~Pb}$, the previously widely used reference. This lead nitrate solution has a chemical shift of $-2960 \mathrm{ppm}$ relative to $\left(\mathrm{CH}_{3}\right)_{4} \mathrm{~Pb}$. Bismuth chemical shifts were referenced to a saturated solution of $\mathrm{Bi}\left(\mathrm{NO}_{3}\right)_{3} \cdot 5 \mathrm{H}_{2} \mathrm{O}$ in concentrated $\mathrm{HNO}_{3}\left(\delta_{\text {iso }}=0.0 \mathrm{ppm}\right)$. Single $\pi / 2$ pulse experiments were performed in solution for both ${ }^{207} \mathrm{~Pb}$ and ${ }^{209} \mathrm{Bi}$ nuclei.

${ }^{209} \mathrm{Bi}$ solid state NMR spectra were referenced $\mathrm{Bi}\left(\mathrm{NO}_{3}\right)_{2}$ and the spectra were acquired using either the Hahn-echo or solid-echo pulse sequences of the forms $(\pi / 2)_{x}-\tau_{1^{-}}(\pi)_{y}-\tau_{2}$-acq and $(\pi / 2)_{x}-\tau_{1^{-}}-(\pi / 2)_{y}-\tau_{2^{-}}$ acq, respectively, where $\tau$ represents the interpulse delays. For ${ }^{209} \mathrm{Bi}$ Hahn-echo experiments, a central-transition selective $\pi / 2$ pulse width of $1.0 \mu \mathrm{s}\left(v_{1}=50 \mathrm{kHz}\right)$ was applied, with an optimized recycle delay of $0.2 \mathrm{~s}$, and spectral width of $2 \mathrm{MHz}$. In case of ${ }^{207} \mathrm{~Pb} \mathrm{NMR}$, the spectrometer pulse was set to maximize the overlap between excitation and the powder pattern. The $\pi / 2$ pulse width ranged between 2.6 and $3.4 \mu \mathrm{s}$ for the different samples. Shorter pulses were used to increase the width of the excitation for the broader powder patterns. The spectral window was set between $100000 \mathrm{~Hz}$ and $625000 \mathrm{~Hz}$, as needed. The pulse repetition times ranged from 7.5 to $75 \mathrm{~s}$. This was set to optimize the $\mathrm{S} / \mathrm{N}$ ratio. Transients were typically collected for 3 days. Depending on the pulse repetition time, this allowed acquisition of between 2000 and 20000 transients. Depending on the spectral window, 512 to 4096 data points were acquired for each transient. This resulted in a digital resolution between 100 and $500 \mathrm{~Hz}$, which corresponds to between 2 and $8 \mathrm{ppm}$.

Small Angle X-ray Scattering (SAXS) and X-ray Diffraction (XRD). Experiments in wide angle transmission geometry (WAXS) were performed with the Nanostar instrument from Bruker AXS. The nanocrystal-toluene dispersions were measured in sealed glass capillaries of $1.5 \mathrm{~mm}$ diameter. The solvent toluene was measured with the same statistical accuracy, and the corresponding signals were removed from the scattering curves of the samples, before applying the fitting procedures. In the WAXS configuration the XRD patterns were recorded with a sample to detector distance of around $5 \mathrm{~cm}$ with an image plate. This allows diffraction peaks up to a $2 \Theta$ angle of around $85^{\circ}$ to be recorded with the $\mathrm{Cu} \mathrm{K} \alpha \mathrm{X}$-ray wavelength of $1.5418 \AA$. For further analysis of the peak width the detector resolution of this setup was determined using the peak widths of a diffraction standard $\left(\mathrm{Al}_{2} \mathrm{O}_{3}\right.$ powder).

High Resolution Transmission Electron Microscopy. HRTEM was performed on drop-casted $\mathrm{PbS}$ nanocrystals on lacey carbon TEM grids with a FEI TITAN ${ }^{3}$ 80-300 equipped with an image aberration corrector $\left(C_{\mathrm{s}}\right.$-corrector $)$ at $300 \mathrm{kV}$ in high resolution mode.

\section{ASSOCIATED CONTENT}

\section{Supporting Information}

The Supporting Information is available free of charge on the ACS Publications website at DOI: 10.1021/acsnano.6b04721.

Supporting Figures S1-S23 and an explanation of the shape data in Figure S10 (PDF)

\section{AUTHOR INFORMATION}

\section{Corresponding Author}

*E-mail: Wolfgang.Heiss@fau.de.

ORCID

Sergii Yakunin: 0000-0002-6409-0565

Dominik Kriegner: 0000-0001-6961-6581

Wolfgang Heiss: 0000-0003-0430-9550

\section{Notes}

The authors declare no competing financial interest.

\section{ACKNOWLEDGMENTS}

A part of the research was performed at the Energie Campus Nürnberg and supported by funding through the "Aufbruch Bayern" initiative of the state of Bavaria. The authors thank the "Österreichische Forschungsförderungsgesellschaft" FFG for financial support via the project Real Nano (Pr. Nr. 843598). This research is partly financially supported by the Austrian Science Fund (FWF-P28167 and J3317-N27). The NMR spectrometers were acquired in collaboration with the University of South Bohemia (CZ) with financial support from the European Research Council via the EFRE INTERREG IV ETC-AT-CZ program (Project M00146, "RERI-uasb"). N.K. acknowledges support by the European Research Council via the Marie-Sklodowska Curie action Phonsi (H2020-MSCA-ITN-642656).

\section{REFERENCES}

(1) Novák, J.; Schlachetzki, A. Heterostructure Epitaxy and Devices; Springer: The Netherlands, 2014.

(2) Henini, M. Molecular Beam Epitaxy: From Research to Mass Production; Newnes, 2012.

(3) Capper, P.; Mauk, M. Liquid Phase Epitaxy of Electronic, Optical and Optoelectronic Materials; John Wiley \& Sons, 2007; Vol. 21.

(4) Prasanthkumar, S.; Saeki, A.; Seki, S.; Ajayaghosh, A. Solution Phase Epitaxial Self-Assembly and High Charge-Carrier Mobility Nanofibers of Semiconducting Molecular Gelators. J. Am. Chem. Soc. 2010, 132, 8866-8867.

(5) Zhu, Y.; Zhou, Y.; Utama, M. I. B.; de la Mata, M.; Zhao, Y.; Zhang, Q.; Peng, B.; Magen, C.; Arbiol, J.; Xiong, Q. Solution pPhase Van der Waals epitaxy of $\mathrm{ZnO}$ Wire Arrays. Nanoscale 2013, 5, 72427249.

(6) Sciacca, B.; Mann, S. A.; Tichelaar, F. D.; Zandbergen, H. W.; Van Huis, M. A.; Garnett, E. C. Solution-Phase Epitaxial Growth of Quasi-Monocrystalline Cuprous Oxide on Metal Nanowires. Nano Lett. 2014, 14, 5891-5898.

(7) Huang, X.; Zeng, Z.; Bao, S.; Wang, M.; Qi, X.; Fan, Z.; Zhang, H. Solution-Phase Epitaxial Growth of Noble Metal Nanostructures on Dispersible Single-Layer Molybdenum Disulfide Nanosheets. Nat. Commun. 2013, 4, 1444.

(8) Peng, X.; Schlamp, M. C.; Kadavanich, A. V.; Alivisatos, A. Epitaxial Growth of Highly Luminescent CdSe/CdS Core/Shell Nanocrystals With Photostability and Electronic Accessibility. J. Am. Chem. Soc. 1997, 119, 7019-7029.

(9) Li, J. J.; Wang, Y. A.; Guo, W.; Keay, J. C.; Mishima, T. D.; Johnson, M. B.; Peng, X. Large-Scale Synthesis of Nearly Monodisperse CdSe/CdS Core/Shell Nanocrystals Using Air-Stable 
Reagents via Successive Ion Layer Adsorption and Reaction. J. Am. Chem. Soc. 2003, 125, 12567-12575.

(10) Reiss, P.; Protiere, M.; Li, L. Core/Shell Semiconductor Nanocrystals. Small 2009, 5, 154-168.

(11) Talapin, D. V.; Mekis, I.; Götzinger, S.; Kornowski, A.; Benson, O.; Weller, H. CdSe/CdS/ZnS and CdSe/ZnSe/ZnS Core-Shell-Shell Nanocrystals. J. Phys. Chem. B 2004, 108, 18826-18831.

(12) Chen, O.; Zhao, J.; Chauhan, V. P.; Cui, J.; Wong, C.; Harris, D. K.; Wei, H.; Han, H.-S.; Fukumura, D.; Jain, R. K.; Bawendi, M. G. Compact High-Quality CdSe-CdS Core-Shell Nanocrystals With Narrow Emission Linewidths and Suppressed Blinking. Nat. Mater. 2013, 12, 445-451.

(13) Dabbousi, B.; Rodriguez-Viejo, J.; Mikulec, F. V.; Heine, J.; Mattoussi, H.; Ober, R.; Jensen, K.; Bawendi, M. (CdSe)ZnS CoreShell Quantum Dots: Synthesis and Characterization of a Size Series of Highly Luminescent Nanocrystallites. J. Phys. Chem. B 1997, 101, 9463-9475.

(14) Xie, R.; Kolb, U.; Li, J.; Basché, T.; Mews, A. Synthesis and Characterization of Highly Luminescent CdSe-Core CdS $/ \mathrm{Zn}_{0.5} \mathrm{Cd}_{0.5} \mathrm{~S} /$ ZnS Multishell Nanocrystals. J. Am. Chem. Soc. 2005, 127, 7480-7488.

(15) Chen, Y.; Vela, J.; Htoon, H.; Casson, J. L.; Werder, D. J.; Bussian, D. A.; Klimov, V. I.; Hollingsworth, J. A. Giant” Multishell CdSe Nanocrystal Quantum Dots With Suppressed Blinking. J. Am. Chem. Soc. 2008, 130, 5026-5027.

(16) Dorfs, D.; Eychmüller, A. Multishell Semiconductor Nanocrystals. Z. Phys. Chem. 2006, 220, 1539-1552.

(17) Cirillo, M.; Aubert, T.; Gomes, R.; Van Deun, R.; Emplit, P.; Biermann, A.; Lange, H.; Thomsen, C.; Brainis, E.; Hens, Z. Flash" Synthesis of CdSe/CdS Core-Shell Quantum Dots. Chem. Mater. 2014, 26, 1154-1160.

(18) Lifshitz, E.; Brumer, M.; Kigel, A.; Sashchiuk, A.; Bashouti, M.; Sirota, M.; Galun, E.; Burshtein, Z.; Le Quang, A.; Ledoux-Rak, I.; Zyss, J. Air-Stable $\mathrm{PbSe} / \mathrm{PbS}$ and $\mathrm{PbSe} / \mathrm{PbSe}_{\mathrm{x}} \mathrm{S}_{1-\mathrm{x}}$ Core-Shell Nanocrystal Quantum Dots and Their Applications. J. Phys. Chem. B 2006, 110, 25356-25365.

(19) Brumer, M.; Kigel, A.; Amirav, L.; Sashchiuk, A.; Solomesch, O.; Tessler, N.; Lifshitz, E. PbSe/PbS and $\mathrm{PbSe} / \mathrm{PbSe}_{\mathrm{x}} \mathrm{S}_{1-\mathrm{x}}$ Core/Shell Nanocrystals. Adv. Funct. Mater. 2005, 15, 1111-1116.

(20) Mekis, I.; Talapin, D. V.; Kornowski, A.; Haase, M.; Weller, H. One-Pot Synthesis of Highly Luminescent CdSe/CdS Core-Shell Nanocrystals via Organometallic and "Greener" Chemical Approaches. J. Phys. Chem. B 2003, 107, 7454-7462.

(21) Cihan, A. F.; Kelestemur, Y.; Guzelturk, B.; Yerli, O.; Kurum, U.; Yaglioglu, H. G.; Elmali, A.; Demir, H. V. Attractive versus Repulsive Excitonic Interactions of Colloidal Quantum Dots Control Blue-to Red-Shifting (and non-Shifting) Amplified Spontaneous Emission. J. Phys. Chem. Lett. 2013, 4, 4146-4152.

(22) Saba, M.; Minniberger, S.; Quochi, F.; Roither, J.; Marceddu, M.; Gocalinska, A.; Kovalenko, M. V.; Talapin, D. V.; Heiss, W.; Mura, A.; Bongiovanni, G. Exciton-Exciton Interaction and Optical Gain in Colloidal CdSe/CdS Dot/Rod Nanocrystals. Adv. Mater. 2009, 21, 4942-4946.

(23) Galland, C.; Ghosh, Y.; Steinbrück, A.; Hollingsworth, J. A.; Htoon, H.; Klimov, V. I. Lifetime Blinking in Nonblinking Nanocrystal Quantum Dots. Nat. Commun. 2012, 3, 908.

(24) Rabouw, F. T.; Vaxenburg, R.; Bakulin, A. A.; van Dijk-Moes, R. J. A.; Bakker, H. J.; Rodina, A.; Lifshitz, E.; Efros, A. L.; Koenderink, A. F.; Vanmaekelbergh, D. Dynamics of Intraband and Interband Auger Processes in Colloidal Core-Shell Quantum Dots. ACS Nano 2015, 9, 10366-10376.

(25) Vaxenburg, R.; Rodina, A.; Lifshitz, E.; Efros, A. L. Biexciton Auger Recombination in $\mathrm{CdSe} / \mathrm{CdS}$ Core/Shell Semiconductor Nanocrystals. Nano Lett. 2016, 16, 2503-2511.

(26) Ning, Z.; Gong, X.; Comin, R.; Walters, G.; Fan, F.; Voznyy, O.; Yassitepe, E.; Buin, A.; Hoogland, S.; Sargent, E. H. Quantum-Dot-inPerovskite Solids. Nature 2015, 523, 324-328.

(27) Gong, X.; Yang, Z.; Walters, G.; Comin, R.; Ning, Z.; Beauregard, E.; Adinolfi, V.; Voznyy, O.; Sargent, E. H. Highly
Efficient Quantum Dot Near-Infrared Light-Emitting Diodes. Nat. Photonics 2016, 10, 253-257.

(28) Wu, L.-M.; Wu, X.-T.; Chen, L. Structural Overview and Structure-Property Relationships of Iodoplumbate and Iodobismuthate. Coord. Chem. Rev. 2009, 253, 2787-2804.

(29) Hirasawa, M.; Ishihara, T.; Goto, T. Exciton Features in 0-, 2-, and 3-Dimensional Networks of $\left[\mathrm{PbI}_{6}\right]^{4-}$ Octahedra. J. Phys. Soc. Jpn. 1994, 63, 3870-3879.

(30) Lindsjö, M.; Fischer, A.; Kloo, L. Anionic Diversity in Iodobismuthate Chemistry. Z. Anorg. Allg. Chem. 2005, 631, 14971501.

(31) Lalancette, R. A.; Elliott, N.; Bernal, I. Crystal Structures and Magnetic Properties of $\mathrm{A}_{2} \mathrm{MnCl}_{6}$ Salts $\left(\mathrm{A}=\mathrm{NH}_{4}^{+}, \mathrm{K}^{+}\right.$and $\left.\mathrm{Rb}^{+}\right)$. J. Cryst. Mol. Struct. 1972, 2, 143-149.

(32) Contreras, J.; Einstein, F.; Gilbert, M.; Tuck, D. Trimethylammonium Hexachloroindate (III). Acta Crystallogr., Sect. B: Struct. Crystallogr. Cryst. Chem. 1977, 33, 1648-1650.

(33) Hines, M. A.; Scholes, G. D. Colloidal PbS Nanocrystals With Size-Tunable Near-Infrared Emission: Observation of Post-Synthesis Self-Narrowing of the Particle Size Distribution. Adv. Mater. 2003, 15, 1844-1849.

(34) Koh, W.-k.; Saudari, S. R.; Fafarman, A. T.; Kagan, C. R.; Murray, C. B. Thiocyanate-Capped PbS Nanocubes: Ambipolar Transport Enables Quantum Dot Based Circuits on a Flexible Substrate. Nano Lett. 2011, 11, 4764-4767.

(35) Hetsch, F.; Zhao, N.; Kershaw, S. V.; Rogach, A. L. Quantum Dot Field Effect Transistors. Mater. Today 2013, 16, 312-325.

(36) Lee, J.-S.; Kovalenko, M. V.; Huang, J.; Chung, D. S.; Talapin, D. V. Band-Like Transport, High Electron Mobility and High Photoconductivity in All-Inorganic Nanocrystal Arrays. Nat. Nanotechnol. 2011, 6, 348-352.

(37) Konstantatos, G.; Howard, I.; Fischer, A.; Hoogland, S.; Clifford, J.; Klem, E.; Levina, L.; Sargent, E. H. Ultrasensitive SolutionCast Quantum Dot Photodetectors. Nature 2006, 442, 180-183.

(38) Boles, M. A.; Ling, D.; Hyeon, T.; Talapin, D. V. The Surface Science of Nanocrystals. Nat. Mater. 2016, 15, 141-153.

(39) Lokteva, I.; Radychev, N.; Witt, F.; Borchert, H.; Parisi, J. r.; Kolny-Olesiak, J. Surface Treatment of CdSe Nanoparticles for Application in Hybrid Solar Cells: the Effect of Multiple Ligand Exchange With Pyridine. J. Phys. Chem. C 2010, 114, 12784-12791.

(40) Webber, D. H.; Brutchey, R. L. Ligand Exchange on Colloidal CdSe Nanocrystals Using Thermally Labile tert-Butylthiol for Improved Photocurrent in Nanocrystal Films. J. Am. Chem. Soc. 2011, 134, 1085-1092.

(41) Mcdonald, S. A.; Konstantatos, G.; Zhang, S.; Cyr, P. W.; Klem, E. J.; Levina, L.; Sargent, E. H. Solution-Processed PbS Quantum Dot Infrared Photodetectors and Photovoltaics. Nat. Mater. 2005, 4, 138142.

(42) Piliego, C.; Protesescu, L.; Bisri, S. Z.; Kovalenko, M. V.; Loi, M. A. 5.2\% Efficient PbS Nanocrystal Schottky Solar Cells. Energy Environ. Sci. 2013, 6, 3054-3059.

(43) Dong, A.; Ye, X.; Chen, J.; Kang, Y.; Gordon, T.; Kikkawa, J. M.; Murray, C. B. A Generalized Ligand-Exchange Strategy Enabling Sequential Surface Functionalization of Colloidal Nanocrystals. J. Am. Chem. Soc. 2011, 133, 998-1006.

(44) Fafarman, A. T.; Koh, W.-k.; Diroll, B. T.; Kim, D. K.; Ko, D.-K.; Oh, S. J.; Ye, X.; Doan-Nguyen, V.; Crump, M. R.; Reifsnyder, D. C.; Murray, C. B.; Kagan, C. R. Thiocyanate-Capped Nanocrystal Colloids: Vibrational Reporter of Surface Chemistry and SolutionBased Route to Enhanced Coupling in Nanocrystal Solids. J. Am. Chem. Soc. 2011, 133, 15753-15761.

(45) Dirin, D. N.; Dreyfuss, S.; Bodnarchuk, M. I.; Nedelcu, G.; Papagiorgis, P.; Itskos, G.; Kovalenko, M. V. Lead Halide Perovskites and Other Metal Halide Complexes As Inorganic Capping Ligands for Colloidal Nanocrystals. J. Am. Chem. Soc. 2014, 136, 6550-6553.

(46) Zhang, H.; Jang, J.; Liu, W.; Talapin, D. V. Colloidal Nanocrystals with Inorganic Halide, Pseudohalide, and Halometallate Ligands. ACS Nano 2014, 8, 7359-7369. 
(47) Ning, Z.; Dong, H.; Zhang, Q.; Voznyy, O.; Sargent, E. H. Solar Cells Based on Inks of n-Type Colloidal Quantum Dots. ACS Nano 2014, 8, 10321-10327.

(48) Crisp, R. W.; Kroupa, D. M.; Marshall, A. R.; Miller, E. M.; Zhang, J.; Beard, M. C.; Luther, J. M. Metal Halide Solid-State Surface Treatment for High Efficiency PbS and PbSe QD Solar Cells. Sci. Rep. 2015, 5, 9954

(49) Yang, Z.; Janmohamed, A.; Lan, X.; García de Arquer, F. P.; Voznyy, O.; Yassitepe, E.; Kim, G.-H.; Ning, Z.; Gong, X.; Comin, R.; Sargent, E. H. Colloidal Quantum Dot Photovoltaics Enhanced by Perovskite Shelling. Nano Lett. 2015, 15, 7539-7543.

(50) Krautscheid, H.; Vielsack, F. Iodoplumbate mit Vier-und Fünffach Koordinierten $\mathrm{Pb}^{2+}$-Ionen. Z. Anorg. Allg. Chem. 1999, 625, $562-566$.

(51) Bieroń, J.; Pyykkö, P. Nuclear Quadrupole Moments of Bismuth. Phys. Rev. Lett. 2001, 87, 133003.

(52) Lechner, R. T.; Fritz-Popovski, G.; Yarema, M.; Heiss, W.; Hoell, A.; Schülli, T. U.; Primetzhofer, D.; Eibelhuber, M.; Paris, O. Crystal Phase Transitions in the Shell of $\mathrm{PbS} / \mathrm{CdS}$ Core/Shell Nanocrystals Influences Photoluminescence Intensity. Chem. Mater. 2014, 26, 5914-5922.

(53) Burian, M.; Fritz-Popovski, G.; He, M.; Kovalenko, M. V.; Paris, O.; Lechner, R. T. Considerations on the Model-Free Shape Retrieval of Inorganic Nanocrystals from Small-Angle Scattering Data. J. Appl. Crystallogr. 2015, 48, 857-868.

(54) Jain, S.; Harker, A.; Cowley, R. Misfit Strain and Misfit Dislocations in Lattice Mismatched Epitaxial Layers and Other Systems. Philos. Mag. A 1997, 75, 1461-1515.

(55) Jesson, D.; Chen, G.; Chen, K.; Pennycook, S. Self-Limiting Growth of Strained Faceted Islands. Phys. Rev. Lett. 1998, 80, 5156.

(56) LeGoues, F.; Reuter, M.; Tersoff, J.; Hammar, M.; Tromp, R. Cyclic Growth of Strain-Relaxed Islands. Phys. Rev. Lett. 1994, 73, 300.

(57) Ramsdell, L. S. The Crystal Structure of Some Metallic Sulfides; University of Michigan, 1925; Vol. 10.

(58) Mitzi, D. B. Organic-Inorganic Perovskites Containing Trivalent Metal Halide Layers: The Templating Influence of the Organic Cation Layer. Inorg. Chem. 2000, 39, 6107-6113.

(59) Krautscheid, H.; Vielsack, F. $\left[\mathrm{Pb}_{18} \mathrm{I}_{44}\right]^{8-}-$ An Iodoplumbate with an Unusual Structure. Angew. Chem., Int. Ed. Engl. 1995, 34, 20352037.

(60) Krautscheid, H.; Vielsack, F.; Klaassen, N. Polymere Iodoplumbate-Synthese und Kristallstrukturen von $\left(\mathrm{Pr}_{3} \mathrm{~N}-\mathrm{C}_{2} \mathrm{H}_{4}-\right.$ $\left.\mathrm{NPr}_{3}\right)\left[\mathrm{Pb}_{6} \mathrm{I}_{14}(\mathrm{dmf})_{2}\right] \cdot 4 \mathrm{DMF}, \quad\left(\mathrm{Pr}_{3} \mathrm{~N}-\mathrm{C}_{2} \mathrm{H}_{4}-\mathrm{NPr}_{3}\right)\left[\mathrm{Pb}(\mathrm{dmf})_{6}\right]-$ $\left[\mathrm{Pb}_{5} \mathrm{I}_{14}\right] \cdot \mathrm{DMF}$ und $\left(\mathrm{Me}_{3} \mathrm{~N}-\mathrm{C}_{2} \mathrm{H}_{4}-\mathrm{NMe}_{3}\right)_{2}\left[\mathrm{~Pb}_{2} \mathrm{I}_{7}\right]$ I. Z. Anorg. Allg. Chem. 1998, 624, 807-812.

(61) Krautscheid, H.; Vielsack, F. Discrete and Polymeric Iodoplumbates with $\mathrm{Pb}_{3} \mathrm{I}_{10}$ Building Blocks: $\left[\mathrm{Pb}_{3} \mathrm{I}_{10}\right]^{4-}, \quad\left[\mathrm{Pb}_{7} \mathrm{I}_{22}\right]^{8-}$, $\left[\mathrm{Pb}_{10} \mathrm{I}_{28}\right]^{8-},{ }^{1 \infty}\left[\mathrm{Pb}_{3} \mathrm{I}_{10}\right]^{4-}$ and ${ }^{2 \infty}\left[\mathrm{Pb}_{7} \mathrm{I}_{18}\right]^{4-}$. J. Chem. Soc., Dalton Trans. 1999, 16, 2731-2735.

(62) Krautscheid, H.; Vielsack, F. $\left[\mathrm{BuN}\left(\mathrm{CH}_{2} \mathrm{CH}_{2}\right)_{3} \mathrm{NBu}\right]_{3}\left[\mathrm{~Pb}_{5} \mathrm{I}_{16}\right]$. $4 \mathrm{DMF}$-ein Iodoplumbat mit Nahezu $\mathrm{D}_{5 \mathrm{~h}}$-Symmetrischem Anion. $Z$. Anorg. Allg. Chem. 2000, 626, 3-5.

(63) Chen, L.; Wu, B.-L.; He, X.-Y.; Mi, J.-X. A monoclinic Modification of $\mathrm{K}_{3}\left[\mathrm{InCl}_{6}\right]$. Acta Crystallogr., Sect. E: Struct. Rep. Online 2006, 62, i143-i144.

(64) Maculan, G.; Sheikh, A. D.; Abdelhady, A. L.; Saidaminov, M. I.; Haque, M. A.; Murali, B.; Alarousu, E.; Mohammed, O. F.; Wu, T.; Bakr, O. M. $\mathrm{CH}_{3} \mathrm{NH}_{3} \mathrm{PbCl}_{3}$ Single Crystals: Inverse Temperature Crystallization and Visible-Blind UV-Photodetector. J. Phys. Chem. Lett. 2015, 6, 3781-3786.

(65) Wuister, S. F.; de Mello Donegá, C.; Meijerink, A. Influence of Thiol Capping on the Exciton Luminescence and Decay Kinetics of CdTe and CdSe Quantum Dots. J. Phys. Chem. B 2004, 108, 1739317397.

(66) Szendrei, K.; Jarzab, D.; Yarema, M.; Sytnyk, M.; Pichler, S.; Hummelen, J. C.; Heiss, W.; Loi, M. A. Surface Modification of Semiconductor Nanocrystals by a Methanofullerene Carboxylic Acid. J. Mater. Chem. 2010, 20, 8470-8473.
(67) Dou, L.; Yang, Y. M.; You, J.; Hong, Z.; Chang, W.-H.; Li, G.; Yang, Y. Solution-Processed Hybrid Perovskite Photodetectors with High Detectivity. Nat. Commun. 2014, 5, 5404.

(68) Sutherland, B. R.; Johnston, A. K.; Ip, A. H.; Xu, J.; Adinolfi, V.; Kanjanaboos, P.; Sargent, E. H. Sensitive, Fast, and Stable Perovskite Photodetectors Exploiting Interface Engineering. ACS Photonics 2015, 2, 1117-1123.

(69) Yakunin, S.; Sytnyk, M.; Kriegner, D.; Shrestha, S.; Richter, M.; Matt, G. J.; Azimi, H.; Brabec, C. J.; Stangl, J.; Kovalenko, M. V.; Heiss, W. Detection of X-ray Photons by Solution-Processed Lead Halide Perovskites. Nat. Photonics 2015, 9, 444-449.

(70) Dondapati, H.; Ha, D.; Pradhan, A. Enhanced Photocurrent in Solution Processed Electronically Coupled CdSe Nanocrystals Thin Films. Appl. Phys. Lett. 2013, 103, 121114.

(71) Remacle, F. On Electronic Properties of Assemblies of Quantum Nanodots. J. Phys. Chem. A 2000, 104, 4739-4747.

(72) Cho, H. Y.; Lee, J. H.; Kwon, Y. K.; Moon, J. Y.; Lee, C. S. Measurement of the Drift Mobilities and the Mobility-Lifetime Products of Charge Carriers in a CdZnTe Crystal by Using a Transient Pulse Technique. J. Instrum. 2011, 6, C01025.

(73) Yakunin, S.; Dirin, D. N.; Protesescu, L.; Sytnyk, M.; Tollabimazraehno, S.; Humer, M.; Hackl, F.; Fromherz, T.; Bodnarchuk, M. I.; Kovalenko, M. V.; Heiss, W. High Infrared Photoconductivity in Films of Arsenic-Sulfide-Encapsulated LeadSulfide Nanocrystals. ACS Nano 2014, 8, 12883-12894. 